\title{
Drug-resistant Hypertension: Is Renal Sympathetic Denervation the Answer?
}

\author{
Keith A. Thompson • Saibal Kar • Raj Makkar • \\ Ronald G. Victor
}

Published online: 19 January 2011

(C) Springer Science+Business Media, LLC 2010

Trial: Symplicity HTN-2 Investigators, Esler MD, Krum $\mathrm{H}$, et al.: Renal sympathetic denervation in patients with treatment-resistant hypertension (The Symplicity HTN2 Trial): a randomized controlled trial. Lancet 2010, 376:1903-1909.

Rating: ••Of major importance.

Introduction: Sympathetic overactivity is thought to be a major contributor to the pathogenesis and progression of human hypertension [1, 2]. In particular, renal sympathetic activation results in renal vasoconstriction, increased renin secretion, and enhanced sodium and water reabsorption, all of which contribute to the development of hypertension [3]. Despite this mechanistic understanding, attempts at modulating sympathetic tone with antiadrenergic drug therapy have been limited by their poor clinical performance and undesirable side-effect profile $[1,4]$. Additionally, historic surgical approaches for the treatment of hypertension with renal sympathectomy have long been abandoned due to a high perioperative morbidity and mortality $[5,6]$. In recent years, the advent of a catheter-based technique using

K. A. Thompson · S. Kar • R. Makkar • R. G. Victor $(\bowtie)$

The Cedars-Sinai Heart Institute,

8700 Beverly Boulevard, South Tower Room 5722,

Los Angeles, CA 90048, USA

e-mail: Ronald.victor@cshs.org

K. A. Thompson

e-mail: Keith.Thompson@cshs.org

S. Kar

e-mail: Karsk@cshs.org

R. Makkar

e-mail: Raj.makkar@cshs.org radiofrequency to destroy the renal nerves has revitalized these long abandoned thoughts of treating hypertension with renal denervation. Following encouraging results of an uncontrolled feasibility trial and a case report [7, 8], a recent randomized controlled trial (Symplicity HTN-2 [Renal Denervation in Patients With Uncontrolled Hypertension]) has further demonstrated the potential for catheter-based renal denervation in the treatment of drug-resistant hypertension.

Aims: The Symplicity HTN-2 trial was designed to determine the safety and effectiveness of catheter-based renal artery denervation with the Symplicity Catheter System (Ardian, Palo Alto, CA) in reducing blood pressure in patients with drug-resistant hypertension.

Methods: In this multicenter trial (involving 24 centers in Europe, Australia, and New Zealand), 106 patients ages 18 to 85 years with a systolic blood pressure of $160 \mathrm{~mm} \mathrm{Hg}$ or more ( $\geq 150 \mathrm{~mm} \mathrm{Hg}$ in patients with type 2 diabetes), despite treatment with three or more antihypertensive medications were randomized to undergo renal denervation while continuing prior drug therapy or to continue prior drug therapy alone. Patients who met initial screening criteria were subsequently excluded from the trial if their blood pressure fell below eligibility criteria at a second clinic visit after a 2 -week screening phase. During this phase, patients were required to document medication compliance and twice-daily home blood pressure monitoring. Patients were also excluded if they were found to have unfavorable renal artery anatomy on imaging; other exclusion criteria included an estimated glomerular filtration rate of less than $45 \mathrm{~mL} / \mathrm{min} / 1.73 \mathrm{~m}^{2}$ and type 1 diabetes. The trial's primary end point was the change from baseline in seated office-based measurement of systolic blood pressure at 6 months. 
During the intervention, the catheter was advanced into the renal arteries and four to six discrete low-power radiofrequency treatments were applied along the length of both renal arteries. Background use of antihypertensive drugs was held constant for the duration of the trial in both groups.

Results: At baseline, the groups were well matched on most characteristics: office blood pressure was $178 / 96 \mathrm{~mm} \mathrm{Hg}$ in the intervention group $(n=52)$ and $178 / 97 \mathrm{~mm} \mathrm{Hg}$ in the control group $(n=54)$. Office-based blood pressure fell by $32 / 12 \mathrm{~mm} \mathrm{Hg}(\mathrm{SD} 23 / 11 ; P<0.001)$ in the patients who underwent renal denervation versus no change in the control group $(1 / 0 \mathrm{~mm} \mathrm{Hg}, \mathrm{SD} 21 / 10 ; P=\mathrm{NS})$. Home blood pressure fell by $20 / 12 \mathrm{~mm} \mathrm{Hg}$ (SD 17/11) in the renal denervation group $(n=32)$ versus no change in the control group $(n=40)$. Twenty-four-hour ambulatory blood pressure fell by 11/ $7 \mathrm{~mm} \mathrm{Hg}(\mathrm{SD} 15 / 11 ; P<0.006)$ in the renal denervation group $(n=20)$ versus no change in the control group $(n=25)$. There were no serious procedural complications in the denervation group and no between-group differences in renal function or in the incidence of adverse events.

Discussion: The authors concluded that renal artery denervation is safe and effective in reducing office blood pressure, home blood pressure, and 24-h blood pressure at 6 months in patients with drug-resistant hypertension.

\section{Comments}

The Symplicity HTN-2 trial is the first randomized controlled trial evaluating catheter-based renal denervation for the treatment of hypertension. The results demonstrate a dramatic reduction in blood pressure in patients with severe drug-resistant hypertension. Thus, Symplicity HTN-2 is a landmark study in the rapidly developing field of invasive device-based therapy for hypertension [1].

The trial has several notable strengths. The research design was both rigorous and innovative, overcoming challenges of research methodology for evaluating devicebased therapy in hypertension [1]. Simply enrolling hypertensive subjects into clinical trials can lead to large nonspecific decreases in blood pressure due to regression to the mean and the Hawthorne effect, the later related to increased medication adherence. The Symplicity HTN-2 investigators effectively eliminated these confounding effects by requiring that blood pressure be elevated on two sequential screening visits before enrollment, and by having patients focus on their blood pressure and medication adherence by giving them home blood pressure monitors and medication $\operatorname{logs}$ between screening visits. Nineteen percent of initially eligible subjects failed repeat screening. That the control group had no change in blood pressure at the end of the 6-month trial speaks to the effectiveness of this clever and powerful design. Blood pressure variability was further reduced by 1) automatic blood pressure monitoring in the office, thus reducing interobserver variability; 2) restricting medication changes during the trial; and 3) using experienced research teams in hypertension centers of excellence to conduct the study.

The observed reduction of blood pressure with renal denervation was substantial. A reduction in office blood pressure of $32 / 12 \mathrm{~mm} \mathrm{Hg}$, if sustained, would be expected to dramatically reduce cardiovascular morbidity and mortality in this high-risk group. The treatment effect was robust as demonstrated by significant decreases in home and ambulatory blood pressure, which more accurately reflect aggregate blood pressure burden on the cardiovascular system and are thus more predictive of hypertensive complications [2].

This study also has several limitations. Nearly $20 \%$ of patients screened for enrollment were ineligible due to unfavorable renal anatomy, placing limits on potential clinical application. The sample size was too small and duration of follow-up too short to permit evaluation of hard clinical outcomes and the potential for reinnervation. Lack of a sham denervation control and lack of blinding to condition assignment are inherent limitations of this type of trial. This limitation notwithstanding, an invasive sham procedure is often logistically difficult to perform and does not ensure adequate blinding of patients and investigators. Only half of the cohort was evaluated by ambulatory blood pressure monitoring, a key research tool especially for evaluating a treatment based on sympathetic mechanisms (which mediate blood pressure surges typified by the "white coat" effect) [2]. The observed reduction in ambulatory blood pressure was one-third the magnitude of the reduction in office blood pressure, suggesting a more conservative estimate of the treatment effect.

The mechanism by which renal artery denervation improves blood pressure control is fascinating and as yet incompletely understood. Although the article's title indicates that the mechanism of the treatment effect is denervation of the renal sympathetic efferent nerve fibers, denervation of renal afferent (sensory) nerve fibers may play an additional role. Activation of renal afferent nerves is thought to trigger central sympathetic activation and contribute to hypertension in the setting of chronic renal failure [9]. The present study raises the intriguing possibility that renal afferent denervation could be the main mechanism of the therapeutic effect in Symplicity HTN-2. A better mechanistic understanding of the observed reduction in blood pressure will require measuring specific effects of radiofrequency ablation on renal sympathetic nerve function - renal blood flow, plasma renin activity, and renal sodium excretion. 
Overall, the results of the Symplicity HTN-2 trial are very exciting and undoubtedly will fuel much more research on invasive device-based antihypertensive therapy not only for drug-resistant primary hypertension but also for mild primary hypertension and secondary hypertension due to chronic renal failure. The public health implications would be staggering if a one-time invasive procedure could replace a lifetime of antihypertensive medication for a fraction of the 1 billion people with hypertension worldwide [10]. In the context of this proof-of-concept trial, renal denervation proved to be an effective adjunct - not a replacement- for multidrug treatment of difficult hypertension. Is radiofrequency ablation a cure for hypertension? Not yet.

\section{Disclosures}

Conflicts of interest: K.A. Thompson: none; S. Kar: has been on the speaker's bureau for Medtronic; R. Makkar: has been on the speaker's bureau for Medtronic; R.G. Victor: has received grants from the following companies: Pfizer, National Institutes of Health, Muscular Dystrophy Association, NicOx, and Novartis; has received research support from the Lincy Foundation and the Donald W. Reynolds Foundation; is on the Advisory Board for St. Jude's and CVRx; has been on the External Advisory Board for Emory University (Cardiology Training Grant program) and the University of Iowa (Clinical Translational Science award); and has been on the speakers' bureau for Pfizer, GlaxoSmithKline, and Novartis.

\section{References}

1. Martin EA and Victor RG: Premise, Promise, and Potential Limitations of Invasive Devices to Treat Hypertension. Curr Cardiol Rep 2010

2. Kaplan NM and Victor RG: Primary Hypertension: Pathogenesis. In Clinical Hypertension 10th Edition. Philadelphia: Lippincott Williams \& Wilkins; 2009: 42-107.

3. DiBona GF: Physiology in perspective: The Wisdom of the Body. Neural control of the kidney. Am J Physiol Regul Integr Comp Physiol 2005, 289:R633-641.

4. Major cardiovascular events in hypertensive patients randomized to doxazosin vs chlorthalidone: the antihypertensive and lipid-lowering treatment to prevent heart attack trial (ALLHAT). ALLHAT Collaborative Research Group. JAMA 2000, 283:1967-1975.

5. Morrissey DM, Brookes VS and Cooke WT: Sympathectomy in the treatment of hypertension; review of 122 cases. Lancet 1953, 1:403-408.

6. Peet MM: Hypertension and its surgical treatment by bilateral supradiaphragmatic splanchnicectomy. Am J Surg 1948, 75:48-68.

7. Krum H, Schlaich M, Whitbourn R, et al.: Catheter-based renal sympathetic denervation for resistant hypertension: a multicentre safety and proof-of-principle cohort study. Lancet 2009, 373:1275-1281.

8. Schlaich MP, Sobotka PA, Krum H, et al.: Renal sympathetic-nerve ablation for uncontrolled hypertension. N Engl J Med 2009, 361:932-934.

9. Converse RL Jr, Jacobsen TN, Toto RD, et al.: Sympathetic overactivity in patients with chronic renal failure. $N \mathrm{Engl} J \mathrm{Med}$ 1992, 327:1912-1918.

10. Doumas $M$ and Douma S: Renal sympathetic denervation: the jury is still out. Lancet 2010, 376:1878-1880. 